\title{
AVALIAÇÃO DA EdE MATTEDI-TAVARES-CASTIER (MTC) PARA A DESCRIÇÃO DE DENSIDADE, PRESSÃO DE VAPOR E VELOCIDADE DO SOM DE HIDROCARBONETOS
}

\author{
D. HOFFMANN ${ }^{1}$, S. MATTEDI ${ }^{1}$, CASTIER, $^{2}{ }^{2}$ e M. PAREDES ${ }^{3}$ \\ ${ }^{1}$ Programa de Pós Graduação em Engenharia Química, Universidade Federal da Bahia \\ ${ }^{2}$ Chemical Engineering Program, Texas A\&M University at Qatar \\ ${ }^{3}$ Instituto de Química - Departamento de Engenharia Química - UERJ \\ E-mail para contato: davih1@yahoo.com.br
}

\begin{abstract}
RESUMO - A economia exige processos cada vez mais eficientes e, para desenvolvê-los, podem-se utilizar modelos, através dos quais é possível descrever o comportamento termodinâmico de fluidos a partir de dados de pressão, volume, temperatura e/ou composição. Este trabalho procura, através da comparação com dados experimentais de densidade, pressão de vapor e velocidade do som em fase líquida, analisar o desempenho da equação de estado Mattedi-Tavares-Castier (MTC), buscando conjuntos de parâmetros e correlações que tornem a EdE capaz de descrever adequadamente todas as propriedades de alcanos lineares consideradas neste trabalho. Os resultados mostram que o melhor conjunto de parâmetros apresenta desvios abaixo de 5,02\%, na mesma ordem de grandeza dos desvios encontrados na literatura. Além disso, os parâmetros $Q, V^{\mathrm{a}}$ e $\mathrm{u}_{00} / \mathrm{R}$ apresentam correlação com o número de carbonos dos respectivos alcanos, sendo possível reduzir a quantidade de parâmetros da equação para dois. Por fim, conclui-se que o termo relativo à energia característica de interação entre moléculas, parâmetro B, é independente da temperatura.
\end{abstract}

\section{INTRODUÇÃO}

A economia exige processos e equipamentos cada vez mais eficientes e a sociedade impõe a prática da sustentabilidade em toda a cadeia industrial. Para desenvolvê-los, é necessária a descrição de propriedades físicas dos fluidos (puros e em misturas) envolvidos. Para tanto, podem-se utilizar equações de estado (EdE), através das quais é possível descrever o comportamento termodinâmico a partir de dados de pressão, volume, temperatura e composição. As equações são originalmente ajustadas para dados de equilíbrio de fases, no entanto é preciso entender também o comportamento de outras propriedades, entre elas densidade e velocidade do som e, normalmente, os modelos falham na descrição destas propriedades para a região de líquido.

Visando a aplicação futura em sistemas de hidrocarbonetos (por exemplo, em sistemas de interesse da indústria do petróleo), foi escolhido um conjunto de alcanos lineares (C1-C20) para avaliar a capacidade da EdE MTC, desenvolvida por Mateddi et al (1998), baseada na teoria de van der Waals generalizada, em representar o comportamento da velocidade do som, pressão de vapor e 


\section{9 a 22 de outubro de 2014 \\ Florianópolis/SC}

densidade de tais substâncias. Primeiramente foram levantados quais os conjuntos de parâmetros que apresentam os melhores resultados, utilizando-se os dados experimentais de n-hexano para, em seguida, aplicá-los aos dados dos outros alcanos estudados. Os resultados obtidos foram comparados com aqueles apresentados por Liang et al (2012), para a EdE PC-SAFT utilizando três parâmetros. Além disso, percebeu-se que alguns parâmetros da EdE MTC apresentavam correlação com o número de carbonos dos alcanos, de forma que o resultado da aplicação de tais correlações aos dados experimentais de C6-C20 foram comparados com os demais resultados.

\section{EQUAÇÃO DE ESTADO MATTEDI-TAVARES-CASTIER (MTC)}

O modelo MTC é uma EdE baseada na teoria de fluido reticulado, cuja função de partição é obtida pela teoria de van der Waals Generalizada, ou seja, pelo produto de uma contribuição atérmica e uma contribuição residual para a energia livre de Helmholtz (Sandler 1985).

A contribuição atérmica, do modelo de retículo de Staverman-Guggenhenim, leva em conta os efeitos entrópicos devido à diferença de tamanho e forma das moléculas. Já a contribuição residual leva em conta as interações entre as moléculas, ou seja, a energia configuracional do fluido.

A EdE MTC apresenta os seguintes parâmetros: Q representa a área superficial da molécula, z representa o número de coordenação da matriz reticular sendo que nesse trabalho se convencionou ser igual a 10 (Mateddi et al 1998), v ${ }^{*}$ representa o volume ocupado por um mol de células, $\mathrm{V}^{\mathrm{a}}$ representa o volume de compactação molar, ou seja, o volume de um mol quando a pressão tende a infinito, $\Psi$ representa a constante característica do retículo, $\mathrm{u}_{00} / \mathrm{R}$ é o parâmetro independente da temperatura da energia característica de interação entre moléculas e B é o parâmetro dependente.

A partir de EdEs, são derivadas equações para diversas propriedades termodinâmicas, como coeficiente de fugacidade (Mateddi et al 1998), Cp (Liang et al 2012), fator de compressibilidade, densidade (Mateddi 1997), e velocidade do som (Paredes et al 2012). Através de dados experimentais, são obtidos os valores dos parâmetros apresentados para cada substância química de interesse.

\section{METODOLOGIA}

\subsection{Dados}

Foram utilizados dois conjuntos de dados, um obtido junto à base de dados do NIST e às correlações do DIPPR (conjunto I) e outro obtido através de uma extensa pesquisa bibliográfica em busca de dados experimentais (conjunto II). 
O conjunto I é formado por dados de pressão de vapor, densidade da fase líquida e velocidade do som na fase líquida na temperatura reduzida entre 0,45 e 0,9 para alcanos lineares de metano $(\mathrm{C} 1)$ a decano (C10). Tal conjunto foi utilizado para: (1) encontrar uma correlação entre parâmetros e o número de átomos de carbono na cadeia; (2) comparar com os resultados obtidos por Liang et al (2012), para a EdE PC-SAFT, utilizando um conjunto de três parâmetros.

O conjunto II é formado por dados de pressão de vapor na temperatura reduzida entre 0,45 e 0,9 obtidos utilizando a correlação do DIPPR e dados de velocidade do som e densidade obtidos na literatura, conforme Tabela 1.

Tabela 1 - Dados Experimentais

\begin{tabular}{|c|c|c|c|c|}
\hline Alcanos & $\begin{array}{c}\text { Faixa de } \\
\text { Temperatura }(\mathrm{K})\end{array}$ & $\begin{array}{c}\text { Faixa de Pressão } \\
(\mathrm{MPa})\end{array}$ & $\begin{array}{c}\text { Número de dados } \\
\text { Densidade }\end{array}$ & $\begin{array}{c}\text { Número de dados Vel. } \\
\text { do Som }\end{array}$ \\
\hline C6 - C20 & $243,16-670,00$ & $0,1-700,0$ & 2267 & 1211 \\
\hline
\end{tabular}

\subsection{Função Objetivo}

Os parâmetros $\mathrm{Q}, \mathrm{v}^{*}, \mathrm{~V}^{\mathrm{a}}, \mathrm{u}_{00} / \mathrm{R}$ e $\mathrm{B}$ foram obtidos por regressão não linear, através da função objetivo apresentada na Equação 1 e dos dados experimentais, utilizando o Solver do software Microsoft Excel 2007.

onde, $\mathrm{P}$ é a pressão de vapor, $\rho$ é a densidade e u é a velocidade do som. Os sobrescritos exp e calc se referem ao valor experimental e calculado, respectivamente, o subscrito i se refere ao ponto, NP, N $\rho$, Nu é o número de pontos de pressão de vapor, de densidade e de velocidade do som respectivamente.

\section{RESULTADOS E DISCUSSÃO}

\subsection{Identificação dos Melhores Conjuntos de Parâmetros}

Tendo em vista as múltiplas opções de conjuntos de parâmetros que podem ser utilizados para descrever o comportamento dos alcanos utilizando a EdE MTC, foram utilizados os dados da literatura de n-hexano para identificar os conjuntos que fornecem os melhores resultados. 
Quanto menor for o valor da função objetivo, melhor é o resultado apresentado pelo conjunto de parâmetros. Foram testados 24 conjuntos sendo que os três melhores são apresentados na Tabela 2. Os conjuntos K, W e Y contêm 4, 4 e 5 parâmetros a serem estimados, respectivamente, incluindo Q e $\mathrm{V}^{\mathrm{a}}$. Percebe-se que os conjuntos $\mathrm{K}, \mathrm{W}$ e $\mathrm{Y}$ apresentam $\Psi$ igual a 18 e $\mathrm{u}_{00} / \mathrm{R}$ com valor livre.

Tabela 2 - Conjunto de Parâmetros

\begin{tabular}{|c|c|c|c|c|c|}
\hline \multirow{2}{*}{ Conjunto } & \multicolumn{5}{|c|}{ Parâmetros } \\
\cline { 2 - 5 } & $\mathrm{B}(\mathrm{K})$ & $\Psi$ & $\mathrm{v}^{*}\left(\mathrm{~cm}^{3} / \mathrm{mol}\right)$ & $\mathrm{u}_{00} / \mathrm{R}(\mathrm{K})$ & Função Objetivo x 100 \\
\hline $\mathrm{K}$ & livre & 18 & 10 & livre & 0,45 \\
\hline $\mathrm{W}$ & fixo 0 & 18 & livre & livre & 0,37 \\
\hline $\mathrm{Y}$ & livre & 18 & livre & livre & 0,37 \\
\hline
\end{tabular}

\subsection{Comparação com PC-SAFT}

A Tabela 3 e a Tabela 4 apresentam os desvios absolutos médios (\%AAD) de densidade, velocidade do som e pressão de vapor, obtidos através a EdE MTC e utilizando os conjuntos de parâmetros K, W e Y para os conjuntos de dados I e II, respectivamente. Tais resultados são comparados com os resultados obtidos por Liang et al (2012) para a EdE PC-SAFT, utilizando três parâmetros.

Tabela 3 - Desvios Absolutos Médios (\%) do Conjunto I

\begin{tabular}{|c|c|c|c|c|c|c|c|c|c|c|c|c|}
\hline & & $\mathrm{C} 1$ & $\mathrm{C} 2$ & $\mathrm{C} 3$ & $\mathrm{C} 4$ & $\mathrm{C} 5$ & C6 & $\mathrm{C} 7$ & $\mathrm{C} 8$ & $\mathrm{C} 9$ & $\mathrm{C} 10$ & Média \\
\hline \multirow{4}{*}{ 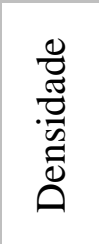 } & Conj. K & 0,37 & 0,40 & 0,95 & 1,20 & 1,48 & 1,83 & 1,65 & 2,02 & 1,35 & 1,40 & 1,26 \\
\hline & Conj. W & 3,29 & 2,49 & 1,58 & 0,82 & 0,79 & 0,77 & 0,61 & 0,82 & 0,50 & 0,51 & 1,22 \\
\hline & Conj. Y & 0,87 & 0,90 & 0,74 & 0,53 & 0,49 & 0,44 & 0,45 & 0,64 & 0,49 & 0,49 & 0,61 \\
\hline & $\mathrm{PC}-\mathrm{SAFT}^{1}$ & 0,83 & 1,75 & 1,90 & 1,80 & 1,59 & 1,49 & 1,63 & 1,56 & 1,82 & 1,59 & 1,60 \\
\hline \multirow{4}{*}{$\begin{array}{l}0 \\
0 \\
0 \\
\frac{0}{0} \\
\frac{\pi}{0} \\
\frac{0}{0} \\
\frac{0}{0} \\
j\end{array}$} & Conj. K & 3,68 & 2,43 & 2,52 & 2,78 & 2,85 & 2,63 & 2,62 & 2,37 & 2,10 & 1,78 & 2,58 \\
\hline & Conj. W & 4,70 & 1,72 & 2,45 & 2,80 & 2,94 & 2,86 & 3,12 & 3,07 & 3,00 & 2,73 & 2,94 \\
\hline & Conj. Y & 3,32 & 1,95 & 2,60 & 3,00 & 3,36 & 3,48 & 3,71 & 3,30 & 3,21 & 2,81 & 3,08 \\
\hline & PC-SAFT $^{1}$ & 1,26 & 0,90 & 1,54 & 1,34 & 1,68 & 0,58 & 1,52 & 0,41 & 2,30 & 1,54 & 1,31 \\
\hline \multirow{4}{*}{ 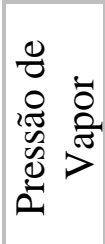 } & Conj. K & 1,23 & 0,38 & 0,73 & 2,02 & 3,68 & 5,21 & 5,67 & 4,67 & 5,30 & 4,76 & 3,36 \\
\hline & Conj. W & 3,37 & 0,76 & 1,23 & 1,64 & 2,73 & 3,35 & 3,25 & 1,64 & 1,86 & 1,07 & 2,09 \\
\hline & Conj. Y & 1,06 & 0,16 & 0,55 & 1,04 & 1,65 & 1,91 & 1,80 & 0,99 & 1,28 & 0,82 & 1,13 \\
\hline & $\mathrm{PC}-\mathrm{SAFT}^{1}$ & 0,52 & 0,68 & 0,65 & 0,72 & 0,58 & 1,74 & 1,13 & 1,16 & 1,40 & 1,28 & 0,99 \\
\hline
\end{tabular}

${ }^{1}$ Liang et al (2012) 
Tabela 4 - Desvios Absolutos Médios (\%) do Conjunto II

\begin{tabular}{|c|c|c|c|c|c|c|c|c|c|}
\hline & & & & & & & & \\
\hline & & C6 & $\mathrm{C} 7$ & $\mathrm{C} 8$ & $\mathrm{C} 9$ & $\mathrm{C} 10$ & $\mathrm{C} 11$ & $\mathrm{C} 12$ & $\mathrm{C} 13$ \\
\hline \multirow{4}{*}{$\begin{array}{l}\frac{0}{0} \\
\frac{\pi}{0} \\
\stackrel{0}{0} \\
0\end{array}$} & Conj. K & 2,38 & 0,49 & 0,69 & 2,50 & 0,98 & 1,39 & 1,09 & 1,55 \\
\hline & Conj. W & 2,96 & 1,25 & 0,68 & 3,25 & 1,24 & 1,93 & 2,40 & 0,62 \\
\hline & Conj. Y & 2,92 & 1,20 & 0,86 & 3,37 & 1,15 & 2,24 & 1,43 & 0,66 \\
\hline & PC-SAFT $^{1}$ & 1,49 & 1,63 & 1,56 & 1,82 & 1,59 & 1,41 & 1,41 & 1,37 \\
\hline \multirow{4}{*}{ 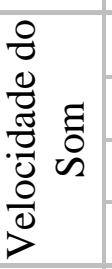 } & Conj. K & 3,41 & 5,47 & 0,84 & 1,19 & 2,96 & 1,29 & 1,32 & 8,76 \\
\hline & Conj. W & 3,94 & 6,70 & 1,08 & 1,79 & 3,98 & 2,17 & 5,66 & 2,14 \\
\hline & Conj. Y & 3,90 & 6,33 & 1,32 & 1,91 & 3,77 & 3,25 & 2,92 & 2,64 \\
\hline & PC-SAFT $^{1}$ & 2,97 & 3,47 & 2,69 & 2,91 & 3,93 & 2,03 & 2,07 & 2,24 \\
\hline \multirow{4}{*}{ 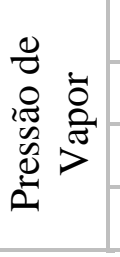 } & Conj. K & 5,29 & 5,32 & 4,79 & 5,41 & 5,05 & 8,68 & 9,08 & 6,54 \\
\hline & Conj. W & 3,62 & 3,02 & 1,70 & 1,93 & 0,83 & 4,36 & 4,44 & 2,24 \\
\hline & Conj. Y & 3,70 & 3,57 & 0,44 & 1,49 & 1,48 & 1,94 & 1,52 & 0,97 \\
\hline & PC-SAFT $^{1}$ & 1,74 & 1,13 & 1,16 & 1,40 & 1,28 & 1,52 & 1,37 & 1,43 \\
\hline \multirow{5}{*}{$\begin{array}{l}\frac{0}{0} \\
\frac{\tilde{J}}{\sqrt[0]{2}} \\
\stackrel{0}{0}\end{array}$} & & $\mathrm{C} 14$ & C15 & C16 & $\mathrm{C} 17$ & C18 & C19 & $\mathrm{C} 20$ & Média \\
\hline & & 0,38 & 1,19 & 0,69 & 0,36 & 2,24 & 1,29 & 0,32 & 1,17 \\
\hline & Conj. W & 2,30 & 0,91 & 0,39 & 1,81 & 1,97 & 2,32 & 1,11 & 1,67 \\
\hline & Conj. Y & 0,71 & 1,00 & 0,60 & 0,67 & 1,95 & 2,40 & 1,21 & 1,49 \\
\hline & PC-SAFT $^{1}$ & 1,37 & 1,37 & 1,33 & 1,29 & 1,28 & 1,26 & 1,24 & 1,43 \\
\hline \multirow{4}{*}{$\begin{array}{l}0 \\
0 \\
0 \\
\frac{\pi}{0} \\
\frac{0}{0} \\
\text { in } \\
\frac{0}{0} \\
>\end{array}$} & Conj. K & 2,26 & 3,76 & 3,36 & 11,11 & 7,29 & 7,12 & 5,34 & 4,37 \\
\hline & Conj. W & 5,41 & 4,59 & 4,94 & 11,41 & 7,66 & 8,09 & 7,66 & 5,15 \\
\hline & Conj. Y & 4,00 & 4,11 & 5,62 & 11,33 & 7,94 & 8,58 & 7,70 & 5,02 \\
\hline & PC-SAFT $^{1}$ & 2,52 & 2,94 & 2,79 & 3,26 & 3,47 & 3,66 & 3,46 & 2,96 \\
\hline \multirow{4}{*}{ 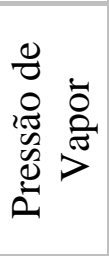 } & Conj. K & 10,96 & 12,37 & 10,93 & 9,24 & 14,20 & 11,44 & 10,15 & 8,63 \\
\hline & Conj. W & 5,83 & 5,97 & 4,32 & 6,62 & 5,02 & 5,85 & 3,24 & 3,93 \\
\hline & Conj. Y & 1,94 & 5,50 & 2,71 & 3,01 & 5,20 & 4,96 & 3,05 & 2,77 \\
\hline & PC-SAFT $^{1}$ & 1,11 & 1,77 & 1,54 & 1,49 & 2,21 & 2,71 & 2,57 & 1,63 \\
\hline
\end{tabular}

${ }^{1}$ Liang et al (2012)

Percebem-se em ambas as tabelas que os valores médios dos desvios (\%AAD) dos três conjuntos são da mesma ordem de grandeza daquele encontrado por Liang et al (2012), com exceção do valor médio de pressão de vapor do conjunto $\mathrm{K}$, que destoou dos demais.

A EdE MTC apresentou desvios (\%AAD) de velocidade do som elevado para os compostos que 
apresentavam uma parcela considerável de dados experimentais em pressões superiores à atmosférica. O caso mais emblemático é para o heptadecano (C17), onde o desvio \%AAD passa de $10 \%$ para os três conjuntos de parâmetros estudados.

Como já era esperado, devido ao resultado apresentado na Tabela 2, os resultados para os conjuntos de parâmetros $\mathrm{Y}$ e W são muito semelhantes, de forma que se pode concluir que o parâmetro B pode ser desprezado para alcanos lineares, ou seja, o termo relativo à energia característica de interação entre moléculas é praticamente independente da temperatura.

\subsection{Correlação de Parâmetros do Conjunto Y}

Paredes et al (1994) verificaram que há correlação entre os parâmetros $\mathrm{u}_{00} / \mathrm{R}, \mathrm{V}^{\mathrm{a}} \mathrm{e} \mathrm{Q}$ e o número de carbono para alcanos lineares. Dessa forma, partindo-se do conjunto de dados I, que são mais comportados, verificou-se que também existe tal correlação nesse trabalho, conforme pode ser notado na Figura 1. Tais correlações foram aplicadas no conjunto de parâmetros Y, reduzindo-o a apenas dois parâmetros. Os valores de $\mathrm{R}^{2}$ para $\mathrm{u}_{00} / \mathrm{R}, \mathrm{V}^{\mathrm{a}}$ e $\mathrm{Q}$ foram $0,998,0,999$ e 0,950 , respectivamente. Os resultados são apresentados na Tabela 5.
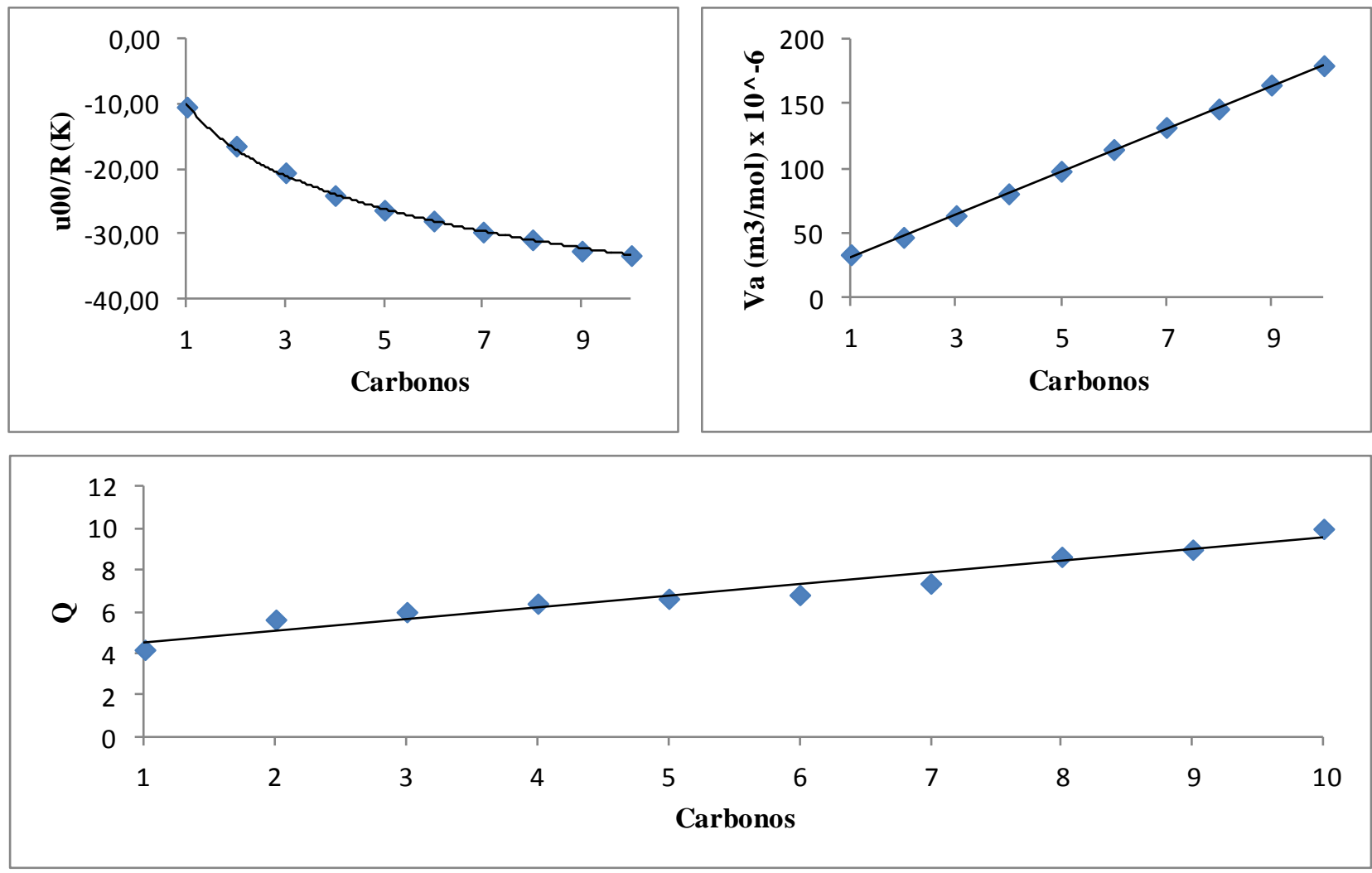

Figura 1- Correlação entre parâmetros $\left(\mathrm{u}_{00} / \mathrm{R}(1 \mathrm{a}), \mathrm{V}^{\mathrm{a}}(1 \mathrm{~b})\right.$ e $\left.\mathrm{Q}(1 \mathrm{c})\right)$ e o número de carbonos

O valor médio dos desvios \%AAD da densidade para os parâmetros correlacionados, apresentados na Tabela 5, consegue ser melhor que aquele obtido pelo conjunto Y, além de ser muito 
parecido com o resultado obtido por Liang et al (2012).

Já em relação à velocidade do som e pressão de vapor, os valores dos desvios do conjunto correlacionado apresenta uma grande diferença para os valores obtidos com o conjunto de parâmetros Y, para ambas as propriedades, a partir do tetradecano (C14). Isso pode ter ocorrido devido à correlação ter sido obtida com dados de $\mathrm{C} 1$ a C10. É possível que, caso sejam geradas correlações utilizando dados de $\mathrm{C} 1$ a C20, os resultados entre dados correlacionados e o conjunto de parâmetros Y sejam muito semelhantes.

Tabela 5 - Desvios Absolutos Médios (\%) para o cálculo de densidade para os dados do Conjunto II com Correlação

\begin{tabular}{|c|c|c|c|c|c|c|c|c|c|}
\hline & & C6 & $\mathrm{C} 7$ & $\mathrm{C} 8$ & $\mathrm{C} 9$ & $\mathrm{C} 10$ & $\mathrm{C} 11$ & $\mathrm{C} 12$ & C13 \\
\hline$\frac{\theta}{0}$ & Correlação & 3,63 & 0,55 & 0,75 & 3,04 & 1,30 & 3,30 & 1,64 & 0,86 \\
\hline$\frac{\sigma}{\Omega}$ & Conj. Y & 2,92 & 1,20 & 0,86 & 3,37 & 1,15 & 2,24 & 1,43 & 0,66 \\
\hline$\stackrel{0}{0}$ & PC-SAFT $^{1}$ & 1,49 & 1,63 & 1,56 & 1,82 & 1,59 & 1,41 & 1,41 & 1,37 \\
\hline$\frac{0}{\sigma} \varepsilon$ & Correlação & 4,67 & 8,07 & 1,23 & 2,22 & 5,53 & 2,61 & 3,91 & 2,79 \\
\hline • & Conj. Y & 3,90 & 6,33 & 1,32 & 1,91 & 3,77 & 3,25 & 2,92 & 2,64 \\
\hline$i^{\infty}$ & PC-SAFT $^{1}$ & 2,97 & 3,47 & 2,69 & 2,91 & 3,93 & 2,03 & 2,07 & 2,24 \\
\hline & Correlação & 2,68 & 2,72 & 0,91 & 1,18 & 2,65 & 3,70 & 4,35 & 2,67 \\
\hline $\begin{array}{ll}\pi & 0 \\
& 0 \\
n & 0\end{array}$ & Conj. Y & 3,70 & 3,57 & 0,44 & 1,49 & 1,48 & 1,94 & 1,52 & 0,97 \\
\hline & PC-SAFT $^{1}$ & 1,74 & 1,13 & 1,16 & 1,40 & 1,28 & 1,52 & 1,37 & 1,43 \\
\hline & & C14 & C15 & $\mathrm{C} 16$ & $\mathrm{C} 17$ & C18 & C19 & $\mathrm{C} 20$ & Média \\
\hline$\frac{0}{0}$ & Correlação & 0,91 & 0,74 & 0,81 & 1,09 & 1,25 & 0,72 & 0,98 & 1,44 \\
\hline$\cdot \stackrel{\overrightarrow{\mathscr{V}}}{=}$ & Conj. Y & 0,71 & 1,00 & 0,60 & 0,67 & 1,95 & 2,40 & 1,21 & 1,49 \\
\hline อั & PC-SAFT $^{1}$ & 1,37 & 1,37 & 1,33 & 1,29 & 1,28 & 1,26 & 1,24 & 1,43 \\
\hline$\frac{\mathscr{\theta}}{\tilde{\tau}} \varepsilon$ & Correlação & 5,39 & 4,29 & 8,43 & 16,11 & 9,33 & 8,95 & 10,28 & 6,25 \\
\hline : & Conj. Y & 4,00 & 4,11 & 5,62 & 11,33 & 7,94 & 8,58 & 7,70 & 5,02 \\
\hline$\sum^{\overline{0}}$ & PC-SAFT $^{1}$ & 2,52 & 2,94 & 2,79 & 3,26 & 3,47 & 3,66 & 3,46 & 2,96 \\
\hline$\ddot{0}$. & Correlação & 7,43 & 10,48 & 9,11 & 9,76 & 13,73 & 14,02 & 15,59 & 6,73 \\
\hline 惢 & Conj. Y & 1,94 & 5,50 & 2,71 & 3,01 & 5,20 & 4,96 & 3,05 & 2,77 \\
\hline 2 & PC-SAFT $^{1}$ & 1,11 & 1,77 & 1,54 & 1,49 & 2,21 & 2,71 & 2,57 & 1,63 \\
\hline
\end{tabular}

${ }^{1}$ Liang et al (2012) 


\section{CONCLUSÃO}

Este trabalho avalia a capacidade da EdE MTC, baseada na teoria de van der Waals generalizada, em representar o comportamento da velocidade do som, pressão de vapor e densidade de alcanos lineares (C1-C20), utilizando dois conjuntos de dados nas faixas de $0,45<\mathrm{Tr}<0,9$ para dados de saturação e $243,16 \mathrm{~K}<\mathrm{T}<670,00 \mathrm{~K}$ e $0,1 \mathrm{MPa}<\mathrm{P}<700 \mathrm{MPa}$ para os demais dados. O melhor conjunto de parâmetros apresenta desvios abaixo de 5,02\%, na mesma ordem de grandeza dos desvios obtidos por Liang et al (2012), para a EdE PC-SAFT utilizando três parâmetros. O parâmetro $\mathrm{B}$ pode ser desprezado para alcanos lineares, ou seja, o termo relativo à energia caracteristica de interação entre moléculas é independente da temperatura. Há correlação entre o número de carbono e o parâmetro independente da temperatura da energia característica de interação entre moléculas $\left(\mathrm{u}_{00} / \mathrm{R}\right)$, o parâmetro relativo ao volume de compactação molar $\left(\mathrm{V}^{\mathrm{a}}\right)$ e o parâmetro relativo à área superficial da molécula $(\mathrm{Q})$. A utilização de tais correlações permite desenvolver a EdE MTC com apenas dois parâmetros.

\section{REFERÊNCIAS}

DIPPR 801 database. Design Institute for Physical Property Data. AIChE, New York, 1998.

LIONG, X.; MOGENSEN, B.M.; THONSEN, K.; YAN, W.; KONTOGEORGIS, G.M. Approach to Improve Speed of Sound Calculation within PC-SAFT Framework. Ind. Eng. Chem. Res., v. 51, p. 14903-14914, 2012.

MATTEDI, S; TAVARES, F.W.; CASTIER, M. Group contribution equation of state based on the lattice fluid theory Alkane-alkanol systems, Fluid Phase Equilibria, v. 142, p. 33-54, 1998.

NIST Chemistry Webbook. http://webbook.nist.gov/chemistry (acessado em janeiro de 2014).

PAREDES, M.L.L.; CARDOSO, A.S.; MATTEDI, S.; TAVARES, F.W.; CASTIER, M. Equação de Estado para Fluidos Polares Polissegmentados. Anais do $10^{\circ}$ Congresso Brasileiro de Engenharia Química, São Paulo, pp. 121-126, 1994.

PAREDES, M.L.L.; REIS, R.A.; SILVA, A.A.; RIBEIRO, M.H.A.; XIMANGO, P.B. Densities, sound velocities, and refractive indexes of (tetralin + n-decane) and thermodynamic modeling by Prigogine-Flory-Patterson model. J. Chem. Thermodynamics, v. 45, p.35-42, 2012.

SANDLER, S.I. The Generalized van der Waals Partition Function. I. Basic Theory. Fluid Phase Equilibria, v. 19, p. 233-257, 1985. 